\title{
(6) OPEN ACCESS \\ Early neonatal vitamin A supplementation and infant mortality: an individual participant data meta-analysis of randomised controlled trials
}

\author{
Neonatal Vitamin A Supplementation Evidence group
}

\begin{abstract}
- Additional material is published online only. To view please visit the journal online (http://dx.doi.org/10.1136/ archdischild-2018-315242)
\end{abstract}

For numbered affiliations see end of article.

Correspondence to Dr Rajiv Bahl, Department of Maternal, Newborn, Child and Adolescent Health, World Health Organization, Geneva 27, Switzerland; bahlr@who.int

Received 29 March 2018 Revised 22 August 2018 Accepted 5 September 2018 Published Online First 13 November 2018

\section{Check for updates}

(c) Author(s) (or their employer(s)) 2019. Re-use permitted under CC BY-NC. No commercial re-use. See rights and permissions. Published by BMJ.

To cite: Neonatal Vitamin A Supplementation Evidence group. Arch Dis Child 2019:104:217-226.

\begin{abstract}
Background Biannual vitamin A supplementation is a well-established survival tool for preschool children 6 months and older in vitamin A deficient populations but this schedule misses the opportunity to intervene on most young infant deaths. Randomised trials of neonatal vitamin A supplementation (NVAS) in the first few days of life to assess its impact on under 6-month mortality in low/middle-income countries have had varying results.

Methods Investigators of 11 published randomised placebo-controlled NVAS trials ( $n=163567$ children)

\section{What is already known on this topic?}

- Vitamin A supplementation of children 6-59 months in settings where the deficiency is a public health problem has been shown to reduce mortality.

- Supplementation between 1 and 5 months has consistently been found as not beneficial.

- Supplementation early in the neonatal period has been observed to reduce mortality in South Asia but not in Africa, for reasons that remain unclear.
\end{abstract} reanalysed their data according to an agreed plan and pooled the primary outcomes of mortality from supplementation through 6 and 12 months of age using random effects models and meta-regression. One investigator withdrew but allowed use of the data. Findings Overall there was no effect of NVAS on infant survival through 6 (risk ratio (RR) $0.97 ; 95 \% \mathrm{Cl}$ 0.89 to 1.06 ) or 12 months of age (RR $1.00 ; 95 \% \mathrm{Cl}$ 0.93 to 1.08) but results varied by study population characteristics. NVAS significantly reduced 6-month mortality among the trials conducted in Southern Asia (RR 0.87; 95\% Cl 0.77 to 0.98), in contexts with moderate or severe vitamin A deficiency (defined as $10 \%$ or higher proportion of women with serum retinol $<0.7$ $\mu \mathrm{mol} / \mathrm{L}$ or $5 \%$ or more women with night blindness) (RR $0.87 ; 95 \%$ Cl 0.80 to 0.94 ), early infant mortality was 30 or more per 1000 live births (RR $0.91 ; 95 \% \mathrm{Cl}$ 0.85 to 0.98$), 75 \%$ or more of infant mortality occurred in the first 6 months of life (RR $0.92 ; 95 \% \mathrm{Cl} 0.84$ to 1.01$)$, or where $>32 \%$ mothers had no schooling (RR 0.88; $95 \% \mathrm{Cl} 0.80$ to 0.96). NVAS did not reduce mortality in the first 6 months of life in trials conducted in Africa, in contexts characterised by a low prevalence of vitamin A deficiency, lower rates of infant mortality and where maternal education was more prevalent. There was a suggestion of increased infant mortality in trials conducted in Africa (RR 1.07; 95\% Cl 1.00 to 1.15). Individual-level characteristics such as sex, birth weight, gestational age and size, age at dosing, parity, time of breast feeding initiation, maternal education and maternal vitamin A supplementation did not modify the impact of NVAS.

Conclusion NVAS reduced infant mortality in South Asia, in contexts where the prevalence of maternal vitamin A deficiency is moderate to severe and early infant mortality is high; but it had no beneficial effect on infant survival in Africa, in contexts where the prevalence of maternal vitamin A deficiency is lower, early infant mortality is low.

\section{What this study adds?}

- Neonatal vitamin A supplementation (NVAS) reduced infant mortality in South Asia, in contexts where prevalence of maternal vitamin A deficiency was of public health importance.

- NVAS reduced mortality where there was high infant mortality and high percentages of mothers never attending school, conditions typical of South Asian study sites.

- No survival benefit, and possibly harm, was suggested in Africa, in contexts where maternal deficiency was milder, infant mortality was lower, maternal schooling was longer.

\section{INTRODUCTION}

Globally, close to 6 million children under 5 died in 2015; the majority of these deaths occurred in the first year of life. ${ }^{1}$ Despite considerable progress in reducing child mortality over the past decade, new interventions are needed to accelerate improvements in child survival.

Maternal and child undernutrition is a major risk factor for child mortality, accounting for nearly half of all under-5 deaths. ${ }^{2}$ Neonatal vitamin A supplementation (NVAS) has been considered as a potential intervention to reduce infant mortality. This expectation was based on the benefits of vitamin A supplementation from 6 months of age in reducing later infant mortality and plausible biological mechanisms by which this could occur, in addition to the observation of widespread maternal vitamin A deficiency (VAD) in some regions of the world. The WHO recommends periodic vitamin A supplementation for children aged 6-59 months. It has been shown to reduce mortality by $12 \%-30 \%{ }^{3-6}$ and 
has been implemented in more than 100 countries worldwide. $^{7}$ However, trials assessing the effect of supplementation in infants aged 1-5 months suggest there is no benefit of supplementation in this age group. ${ }^{8-11}$ Initial randomised trials of NVAS showed mixed results regarding their effects on improving survival. Trials in Indonesia, Bangladesh and India found significant reductions in mortality during the first year of life,${ }^{12-14}$ while a small trial from Nepal, ${ }^{10}$ a trial conducted in Zimbabwe ${ }^{1516}$ and three trials carried out in Guinea-Bissau ${ }^{17-19}$ found no evidence of benefit. Two meta-analyses published in 2011 identified the same seven randomised controlled trials, ${ }^{12-18}$ but reached different conclusions about the effects of NVAS on infant survival. ${ }^{2021}$

The WHO convened a technical consultation in December 2008 to review the existing evidence regarding NVAS. The technical consultation concluded that there was insufficient evidence to inform public health policy. Three additional randomised trials were commissioned following its recommendations. The publication of these large trials conducted in Ghana, India and Tanzania added substantially to the evidence base. While the trial conducted in India found a 10\% reduction in mortality through to 6 months after supplementation, ${ }^{22}$ the trials in Ghana and Tanzania found no survival benefit. ${ }^{2324}$ The results of these trials were reviewed in the context of pre-existing trials at a technical consultation led by the WHO in April 2014. At the consultation, experts agreed that it would be important to pool the data and explore potential reasons for differences in trial results across sites. The trial investigators convened a working group in September 2014 to develop the protocol and complete this meta-analysis. The analysis plan is available on request. Since the meeting, results from a cluster randomised effectiveness trial conducted in Pakistan were published. ${ }^{25}$ The Pakistani investigators provided their data that have been included in the analyses.

The objective of this paper is to present the findings on the efficacy of NVAS in reducing mortality under 6 and 12 months and how its effects may be modified by study population context characteristics in order to inform the development of public health recommendations.

\section{METHODS}

Potential studies for inclusion in the meta-analysis were identified through a systematic review that started with the examination of two published meta-analyses of NVAS. ${ }^{20} 21$ These reviews were updated using the Cochrane Central Register of Controlled Trials (CENTRAL) (The Cochrane Library), EMBASE, MEDLINE, clinical trial websites, conference proceedings, donor agencies, experts and researchers, the same search statement as in Gogia and Sachdev's [21] study for identification of any additional randomised controlled trials published between 15 October 2010 and 22 February 2016. Data from a further randomised controlled trial were included in the analysis after its publication in June $2016 .^{25}$

The primary outcomes were mortality from date of supplementation to 6 and 12 months. The secondary outcome is to examine how its effects may be modified by study-level and individual-level characteristics. The primary and secondary outcomes remain unchanged during the course of the study.

\section{Eligibility criteria and risk of bias assessment}

Individual or cluster randomised trials assessing the effect of early NVAS (25000-50 000 IU intended to be given within the first 2-3 days of life) compared with placebo, with infant follow-up through at least 6 months of age, were eligible for inclusion in the meta-analysis. This excluded the West et al's study as the period of supplementation extended beyond 3 days of life. ${ }^{10}$

Risk of bias in the included studies was assessed following the Cochrane Handbook recommendations.

\section{Data collection process}

Investigators for each of the identified trials (except the Soofi et al's trial ${ }^{25}$ ) met in France in September 2014 to develop a harmonised pooled analysis protocol (not available in public domain), define subgroups of interest and generate trial-specific estimates for each subgroup. The same protocol and definitions were used for the Pakistan trial data. For all trials, effect sizes were provided as relative risks, except for Pakistan for which effects were expressed as ORs.

\section{Statistical methods}

The primary outcomes were mortality from date of supplementation to 6 months and to 12 months.

To ensure comparability between studies, the effect size and corresponding 95\% CIs were non-parametrically calculated using all randomised infants as the denominator for each individually randomised trial. Effect sizes and 95\% CIs were calculated using generalised estimating equation logistic regression model with log link and exchangeable correlation for the cluster randomised trials. Effect sizes were calculated for each study overall and for each of the specified subgroups.

We assessed the magnitude of heterogeneity across studies with $\mathrm{I}^{2}$ statistics and corresponding $\mathrm{p}$ values. We pooled the overall study-specific point estimates using random effects models because of substantial heterogeneity $\left(\mathrm{I}^{2}>50, \mathrm{p}\right.$ for heterogeneity $<0.05$ ) between studies. Subsequent subgroup analyses to explore potential sources of heterogeneity were conducted using fixed effects meta-analysis. We compared subgroups using the $\mathrm{X}^{2}$ statistical test for heterogeneity.

\section{Subgroup analysis}

We performed bivariate meta-regression by study-level characteristics to identify populations where the intervention may have differential effects. We defined 'study level characteristics' as the characteristics of the target populations of the studies. These included geographic region (Africa or Asia); prevalence of maternal VAD which was assessed by study or extant data for a country or region, as there being none $(0 \%-1 \%)$ or mild prevalence $(2 \%-9 \%)$ versus having a moderate $(10 \%-19 \%)$ or severely high $(\geq 20 \%)$ prevalence, adapting cut-offs from previous WHO guidance for assessing VAD in children ${ }^{26} 27$; early infant mortality, that is, mortality in the control group in the first 6 months of life $(<30$ per 1000 enrolled infants or $\geq 30$ per 1000 enrolled infants); ratio of 6 -month mortality to 12 -month mortality in the control group ( $<75 \%$ or $\geq 75 \%$ ); proportion of infants given the first Diphtheria Pertussis Tetanus vaccine (DPT) dose by 6 months $(<93 \%$ or $\geq 93 \%$ ); and proportion of women who have never been to school $(<32 \%$ or $\geq 32 \%)$. Maternal vitamin A moderate or severe status was defined as $10 \%$ or more women with serum retinol $<0.7 \mathrm{mmol} / \mathrm{L}$ or $5 \%$ or more women with night blindness, according to the WHO Global Database on Vitamin A Deficiency. ${ }^{28}$ Thresholds to categorise mortality at 6 months, ratio of 6 -month to 12-month mortality, proportion of infants given first DPT by 6 months and the proportion of women who never attended school were established by using the median of the distribution of the characteristic in the total study population. Meta-analysis and meta-regression were performed in STATA (V.13).

To further investigate potential sources of heterogeneity, we also performed subgroup analyses by a number of individual-level 
characteristics. Subgroups of interest included: infant sex (male or female); birth weight $(<2500$ or $\geq 2500 \mathrm{~g}$ ); gestational age and weight for gestational age categories (preterm small for gestational age, term small for gestational age, preterm average for gestational age, or term average for gestational age), age at vitamin A supplementation ( $<24$ hours, $24-47$ hours or $\geq 48$ hours), parity ( 1 child, $2-3$ children or $\geq 4$ children), maternal education (none, primary school, secondary or more school), time of breast feeding initiation ( $\leq 1$ hour, 2-23 hours or $\geq 24$ hours), colostrum given (yes or no), and maternal largedose vitamin A supplementation (received or not received) and maternal serum retinol $(<1.05 \mu \mathrm{mol} / \mathrm{L}$, indicating low to deficient status or $\geq 1.05 \mu \mathrm{mol} / \mathrm{L}$, indicating adequate status).

\section{Patient involvement}

This manuscript uses data from published studies to address questions identified in consultation with public health researchers, clinicians and nutritionists. In the contributing studies, definition of intervention delivery and data collection opportunities considered patients' practices and preferences. The intervention was delivered at a single contact with the patient and was provided free of cost to families. Prior to study initiation, community leaders and families in the study areas were engaged by the teams that shared information and obtained participants' consent. At the completion of the individual studies, consultations were held to disseminate findings and discuss their implications.

\section{RESULTS}

We included 11 studies from eight countries: Bangladesh, Ghana, Guinea-Bissau (three studies), India (two studies), Indonesia, Pakistan, Tanzania and Zimbabwe that met eligibility criteria. These trials included a total of 163567 infants. Nine were individually randomised, and two were cluster randomised. Six individually randomised trials had two arms, NVAS (48 000-50 000 IU) and placebo. ${ }^{12} 141822-24$ One individually randomised trial in Guinea-Bissau among low birthweight infants had a factorial design and compared NVAS (25 000 IU) with placebo plus early BCG vaccine or on-time BCG vaccine. ${ }^{17} \mathrm{~A}$ parallel, individually randomised trial in Guinea-Bissau among normal birthweight infants was a three-arm trial which compared NVAS (50 $000 \mathrm{IU})$, NVAS (25 $000 \mathrm{IU}$ ) and placebo. ${ }^{19}$ The individually randomised trial in Zimbabwe was a $2 \times 2$ factorial design comparing NVAS (50 $000 \mathrm{IU}$ ), maternal vitamin A supplementation (MVAS; $400000 \mathrm{IU}$ ), NVAS plus maternal supplementation, and placebo; results were published separately for mothers who were HIV positive and HIV negative. ${ }^{15}{ }^{16}$ The trial in Bangladesh was a cluster randomised trial nested within an MVAS trial, and infants were supplemented from birth up to 30 days of age (median age at dosing 7 hours (IQR: 2-18)). ${ }^{13}$ The trial in Pakistan was a cluster randomised trial that compared NVAS (50 $000 \mathrm{IU}$ ) with placebo delivered by local health staff. ${ }^{25}$ All identified published trials that met the intervention definition and reported on the primary outcome of this joint analysis were included. The risk of bias within included studies was determined to be low (figure 1). Notably, half of the included trials published null findings and selective reporting within studies was avoided by pooling the data specifically for purposes of this meta-analysis.

\section{STUDY CHARACTERISTICS}

Study characteristics are summarised in online supplementary appendix A. Online supplementary appendix B provides information on the studies' populations and individual-level prevalence of the effect modifiers examined. Online supplementary appendix $\mathrm{C}$ provides risk ratios per subgroup by site.

\section{RESULTS OF INDIVIDUAL STUDIES}

Four studies found reductions in infant mortality between supplementation with NVAS and 6 months $^{12-14} 22$ and seven studies ${ }^{15-19} 23-25$ found no evidence of reduction. Regarding the effect of NVAS on mortality through 12 months, two studies found reductions in mortality, ${ }^{12}{ }^{13}$ while the remaining eight studies found no evidence of reduction. One trial did not follow-up infants beyond 6 months of age. ${ }^{14}$

\section{SYNTHESIS OF RESULTS}

\section{Overall pooled analysis}

After pooling the 11 trials which reported mortality risk at 6 months, there was no evidence of a reduced risk of death; the pooled risk ratio (RR) based on a random effects model was $0.97 ; 95 \%$ CI 0.89 to 1.06 (table 1 and figure 2).

After combining data from the 10 trials which reported mortality by 12 months, there was also no evidence of a reduced risk of death; the pooled RR calculated with a random effects model was $1.00 ; 95 \%$ CI 0.93 to 1.08 (figure 3 ).

\section{Pooled results stratified by study-level characteristics}

Using meta-regression, we identified five variables as significantly associated with the effect of NVAS on mortality at 6 months: geographic region of study, prevalence of maternal VAD, control group mortality at 6 months, ratio of 6 -month to 12 -month mortality and proportion of mothers with no schooling. Geographic region and prevalence of maternal VAD were associated with the effect of NVAS on mortality by 12 months (table 1 ).

\section{Geographic region}

Based on the five trials conducted in Asia, NVAS was associated with a $13 \%$ lower risk of death until 6 months (RR $0.87 ; 95 \% \mathrm{CI}$ 0.77 to 0.98 ). In Africa, based on six trials, the intervention was associated with higher, but not statistically significant, risk of death (RR 1.06; 95\% CI 0.98 to 1.15). Based on four trials in Asia which followed infants through 12 months, NVAS was associated with a 9\% lower risk of infant death (RR $0.91 ; 95 \%$ CI 0.80 to 1.03 ), but this was not statistically significant. In Africa, based on six trials, NVAS was associated with 7\% higher risk of death through 12 months (RR 1.07; 95\% CI 1.00 to 1.15).

\section{Maternal VAD}

NVAS was associated with $13 \%$ lower mortality to 6 months in the pooled results from three trials conducted in study populations where the prevalence of maternal VAD was moderate/ severe (RR $0.87 ; 95 \%$ CI 0.80 to 0.94 ), but not in the pooled results from seven trials conducted in study populations with no or mild deficiency (RR 1.05; 95\% CI 0.96 to 1.15 ). We observed similarly contrasting results regarding mortality through 12 months. In study populations where the prevalence of maternal VAD was moderate or severe, two trials observed a $9 \%$ reduction in mortality (RR $0.91 ; 95 \% \mathrm{CI} 0.83$ to 1.00 ); in the seven study populations with no or mild maternal deficiency, mortality was $6 \%$ higher but this was not statistically significant (RR 1.06; $95 \%$ CI 0.98 to 1.15 ).

Control group mortality at 6 months and ratio of mortality to 6 and 12 months

Among six trials conducted in study populations where 6-month mortality in the control group was 30 or more per 1000 live 


\begin{tabular}{|c|c|c|c|c|c|c|}
\hline & 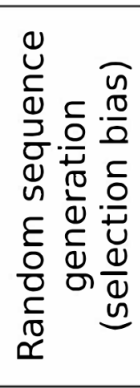 & 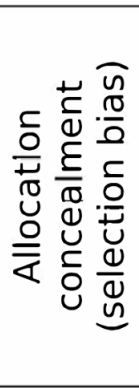 & 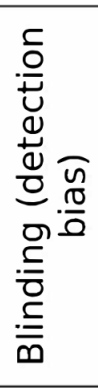 & 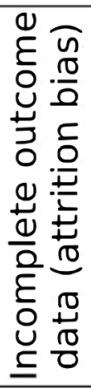 & 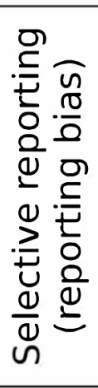 & $\begin{array}{l}n \\
\frac{n}{0} \\
\frac{0}{0} \\
\frac{1}{0} \\
\frac{1}{1} \\
0\end{array}$ \\
\hline Benn (2008) & + & + & + & + & + & + \\
\hline Benn (2010) & + & + & + & + & + & + \\
\hline Benn (2014) & + & + & + & + & + & + \\
\hline Edmond (2014) & + & + & + & + & + & + \\
\hline Humphrey (1996) & + & + & + & + & + & + \\
\hline Klemm (2008) & + & + & + & + & + & + \\
\hline $\begin{array}{l}\text { Malaba } \\
(2005) / \text { Humphrey } \\
(2006)\end{array}$ & + & + & + & + & + & + \\
\hline Masanja (2014) & + & + & + & + & + & + \\
\hline Mazumder (2014) & + & + & + & + & + & + \\
\hline Rahmathullah (2003) & + & + & + & + & + & + \\
\hline Soofi (2016) & + & + & + & + & + & + \\
\hline
\end{tabular}

Figure 1 Summary of methodological quality of included studies ('+' indicates low risk of bias and '-' indicates high risk of bias).

births, we found a $9 \%$ lower risk of death up to 6 months of age with NVAS (RR 0.91,95 CI\% 0.85 to 0.98 ); but we found a non-significant $8 \%$ higher risk of death among the five trials conducted in populations where 6-month mortality in the control group was under 30/1000 (RR $1.08 ; 95 \%$ CI 0.95 to 1.24). Further, among six trials conducted in populations where $75 \%$ or more of infant mortality occurred in the first 6 months, there was evidence of lower mortality associated with NVAS (RR 0.92 ; $95 \%$ CI 0.84 to 1.01 ), but this was not statistically significant. The opposite was seen among four trials conducted in populations where less than $75 \%$ of infant mortality occurred in the first 6 months (RR 1.11; 95\% CI 1.00 to 1.22).

\section{Maternal education}

Among four trials conducted in study populations where the $\geq 32 \%$ of mothers had never been to school, we found a $12 \%$ lower risk of death in the first 6 months of life with NVAS (RR $0.88 ; 95 \% \mathrm{CI} 0.80$ to 0.96 ); but we found no difference in the risk of death among the five trials conducted in populations where $<32 \%$ of mothers had never been to school (RR 1.04; $95 \%$ CI 0.92 to 1.18$)$.

\section{Pooled results stratified by individual-level characteristics}

Meta-analyses of effects of NVAS at 6 and 12 months stratified by 11 individual-level characteristics (sex, birth weight, size for gestation, age at dosing, parity, maternal education, time of breast feeding initiation, colostrum given, MVAS and maternal night blindness) did not show any significant heterogeneity between subgroups. The only exception was stratification by maternal serum retinol at baseline, which showed significant heterogeneity $(p=0.042)$ in effects of NVAS among infants born to mothers with low to deficient (RR $0.73 ; 95 \% \mathrm{CI} 0.51$ to 1.05 ) and adequate status (RR 1.26 ; $95 \% \mathrm{CI} 0.86$ to 1.84 ), thus suggesting effect modification. However, the RR was not significant in either of the two subgroups (table 2).

While there was no indication that size for gestation and maternal night blindness are significant effect modifiers, two individual subgroups by these characteristics showed significant effects. NVAS significantly reduced the risk of death under 6 months among term small for gestational age infants (RR 0.86; $95 \%$ CI 0.77 to 0.97 ) but not among other subgroups by size and gestation in pooled results from six studies. NVAS also significantly reduced risk of death by 6 months in four studies among infants of mothers who reported no night blindness (RR $0.90 ; 95 \%$ CI 0.82 to 0.98 ), but not significantly so in mothers who reported night blindness (RR $0.89 ; 95 \%$ CI 0.64 to 1.26 ) (table 2).

There was no significant benefit or harm identified for any of the subgroups by individual-level characteristics regarding mortality through 12 months (table 2). Online supplementary appendix $\mathrm{C}$ provides information on the estimates of the effects of NVAS on 6 and 12-month mortality for each study in the subgroups.

\section{DISCUSSION}

The pooled effect estimates from all studies indicate that NVAS had no overall benefit in reducing mortality through 6 or 12 months of age. A similar finding was reported by Haider et al in their 2017 systematic review. ${ }^{29}$ However, there was significant heterogeneity, suggesting that the effects of NVAS on survival are influenced by study population characteristics. 


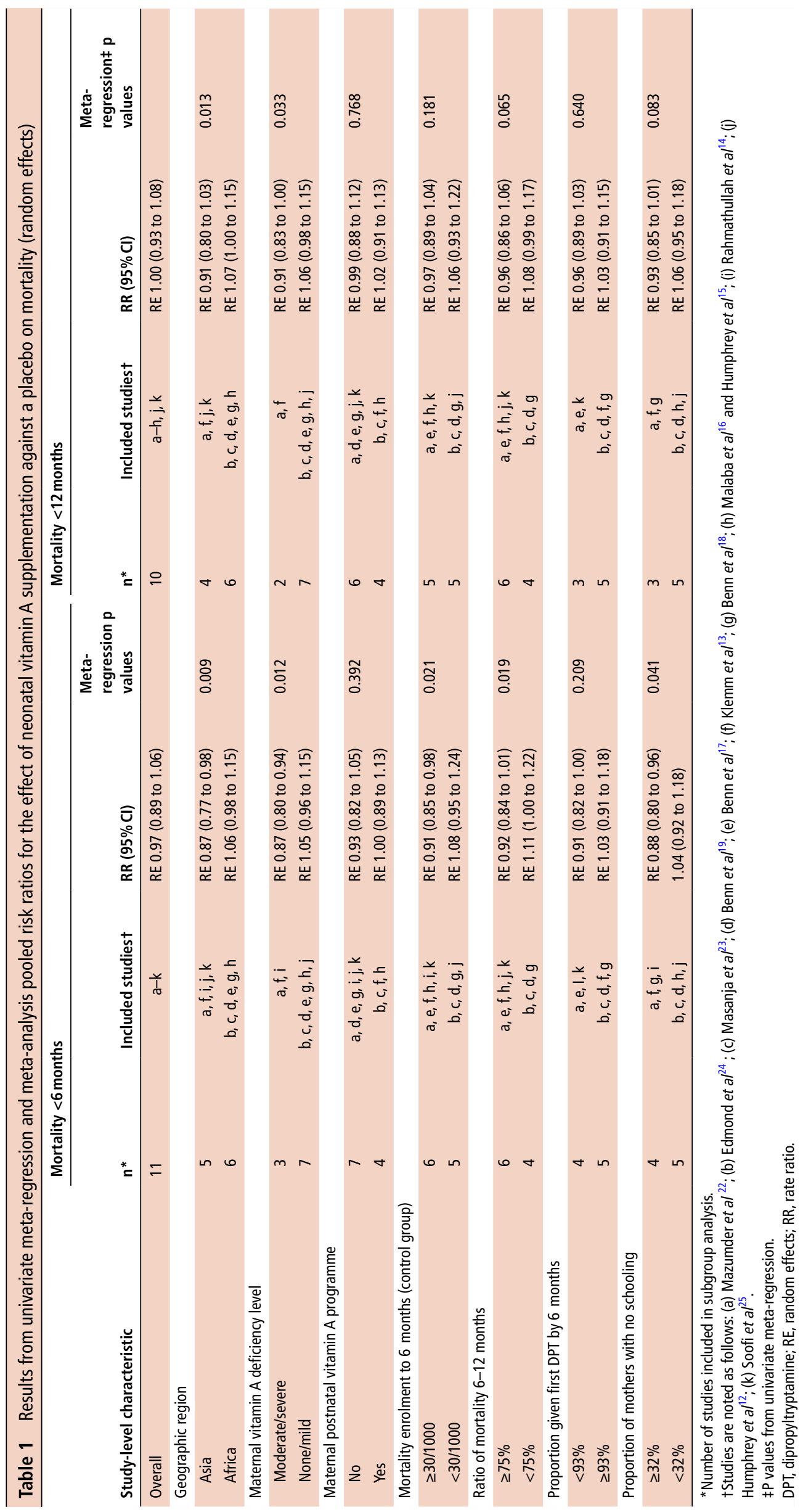




\section{Mortality: randomisation to 6 months}

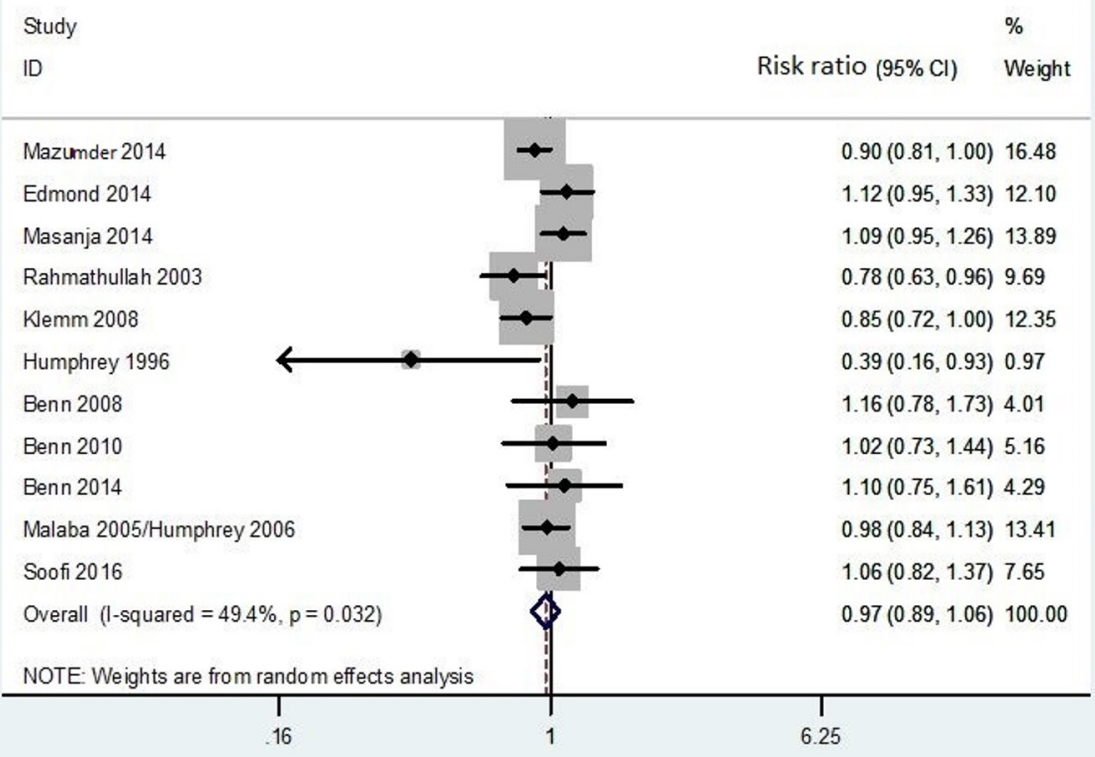

Figure 2 Pooled effect of neonatal vitamin A supplementation (NVAS) on mortality between randomisation and 6 months of age in all studies.

Most studies conducted in Asia showed benefits for survival (RR 0.87 ; $95 \%$ CI 0.77 to 0.98 ) in the first 6 months of life, but no significant effect at 12 months (RR 0.91 ; 95\% CI 0.80 to 1.03). In Africa, however, none of the studies showed benefits, and the overall results could not rule out the possibility of it causing harm (RR $1.06,95 \%$ CI 0.98 to 1.15 for the first 6 months; and RR $1.07,95 \%$ CI 1.00 to 1.15 for the first 12 months).
We propose that geography is a marker of differences in study population contexts. In Asia, studies were conducted in populations with a higher prevalence of maternal VAD, higher early infant mortality, higher proportion of infant deaths within the first 6 months of life and lower levels of maternal schooling. In Africa, on the other hand, study populations lived in contexts that had a lower prevalence of maternal VAD, lower infant mortality, lower early proportion of infant deaths in the first

\section{Mortality: randomisation to 12 months}

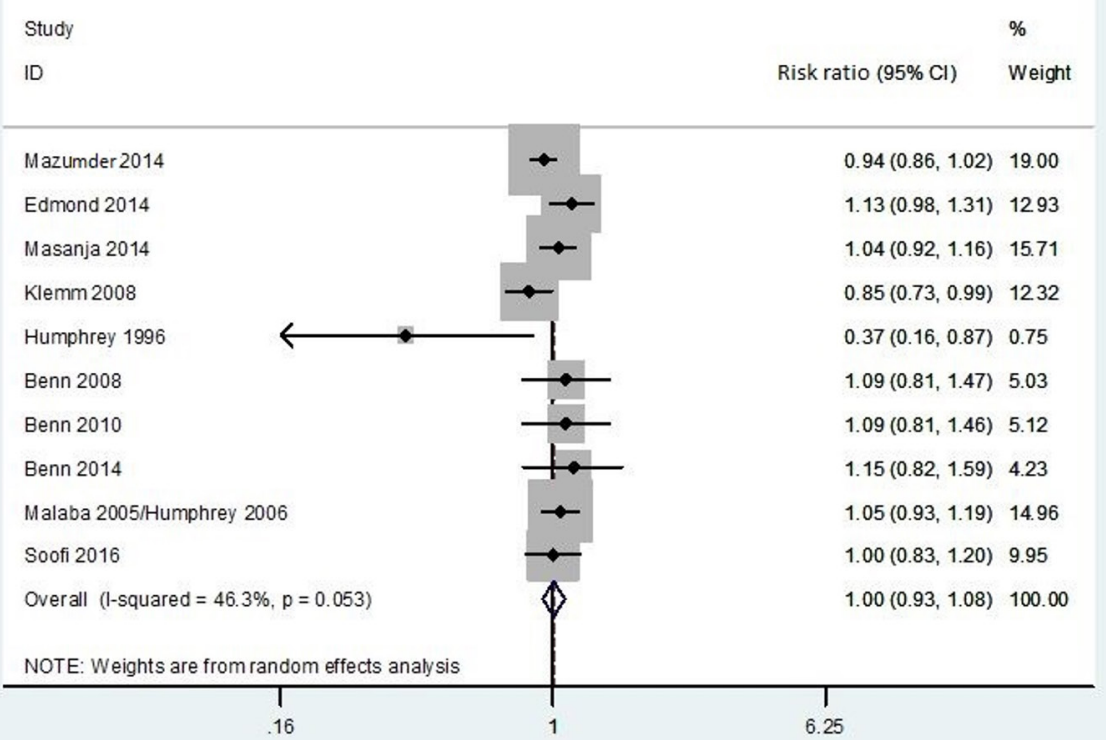

Figure 3 Pooled effect of neonatal vitamin A supplementation (NVAS) on mortality between randomisation and 12 months of age in all studies. 


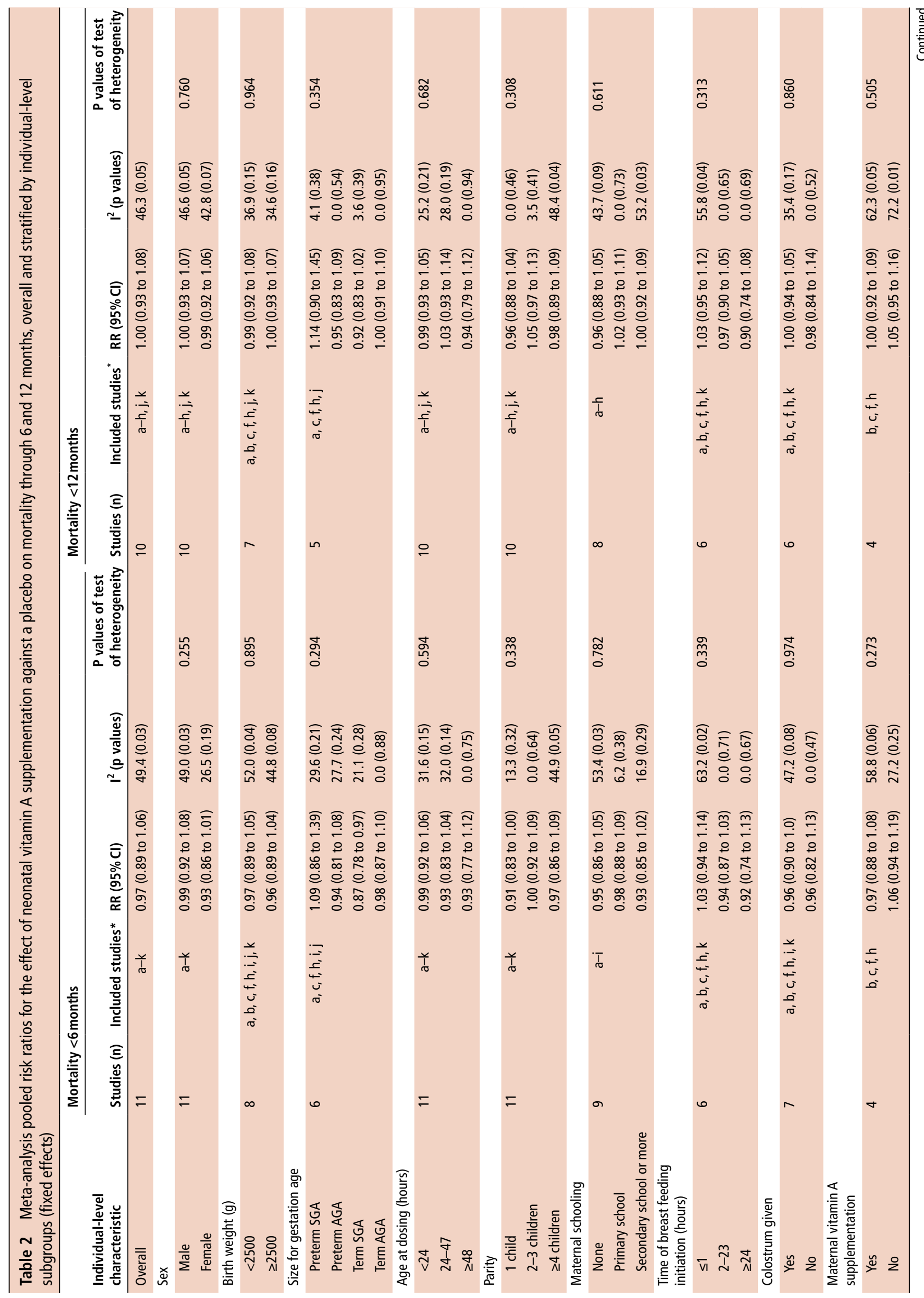


6 months and higher levels of maternal schooling. The differences in observed effects of NVAS between the two continents are likely to reflect, to some extent, the underlying differences in the contexts where populations were studied.

We found that NVAS reduced mortality in populations classified as having a moderate/severely high prevalence of maternal VAD, defined as $10 \%$ or higher proportion of women with serum retinol $<0.7 \mathrm{mmol} / \mathrm{L}$ or $5 \%$ or more women with night blindness. Notably, all the studied populations meeting this prevalence threshold were in Southern Asia. This finding was partially supported by meta-analysis using individual-level characteristics, which suggested that baseline maternal retinol level may be an effect modifier for the effect of NVAS. However, maternal night blindness at individual level did not modify the effect of NVAS, as maternal night blindness was treated when detected during pregnancy in the trials.

We observed that studies conducted in contexts with higher early infant mortality showed a significant benefit of NVAS, an observation already made by Haider and Bhutta in their 2011 systematic review. ${ }^{20}$ The mechanisms underlying this effect of NVAS on mortality are unclear. There are plausible ways through which NVAS could reduce severity of infection and mortality. Neonates in undernourished populations tend to be vitamin A deficient by conventional plasma indicators. ${ }^{30}$ In South India, where newborn vitamin A supplementation lowered mortality by $22 \%,{ }^{14}$ case fatality from diarrhoea and fever was reduced by $40 \%-50 \%$ during the first 6 months, ${ }^{31}$ possibly reflecting antioxidant and anti-inflammatory roles of vitamin A in preserving intestinal tract defenses. ${ }^{32-34}$ In the same study, newborn vitamin A also halved the risk of incident nasopharyngeal pneumococcal colonisation from 2 to 4 months of age, ${ }^{35}$ suggestive of strengthened postnatal respiratory defenses. An alternative mechanism, suggested in published literature, that may explain the differences in Asia and Africa is that NVAS has harmful effects in females after they receive DPT vaccine, and the proportion of infants who receive DPT vaccine on time was higher in African study sites. ${ }^{36}$

Meta-analyses by individual-level characteristics that could be proxies for infant vitamin A status did not support the hypothesis that NVAS efficacy is modified by maternal or newborn baseline vitamin A status. Low birthweight infants and preterm infants in this analysis, either appropriate or small for gestational age, who would have lower vitamin A stores than their normal birth weight and term peers, ${ }^{37} 38$ did not show benefit from NVAS. We acknowledge, nonetheless, that the data on gestational age had limitations. Similarly, we did not find a significant benefit of NVAS among infants whose mothers had not received postpartum MVAS. However, the validity of postpartum MVAS programmes as a proxy for maternal vitamin A status is not clear, as it may depend on a mother's pre-existing level of VAD and quality of programme implementation. In fact, a subgroup analysis among participants in the Tanzania trial found that the effect of NVAS was modified by both postpartum MVAS and the amount of vitamin A that women consumed through food. ${ }^{39}$

We must acknowledge that the associations derived from meta-regressions are observational, and do not have the strength of causal relationships derived from randomised comparisons. This has been noted to apply particularly when averages of patient characteristics in each trial are used as covariates in the regression. We must also acknowledge that we conducted many subgroup analyses, and some of the significant differences between subgroups may have occurred by chance.

There are many strengths of this analysis. We included data from all published trials of NVAS, bringing together information from over 160000 newborns in eight different countries. 
The trial-specific subgroup analysis was conducted by the investigators themselves following a jointly agreed plan on using individual data records. Study-specific relative risks for each subgroup were calculated using the same definitions and methods for every study, ensuring that observed heterogeneity was not due to analytical differences in the way the effect estimates were calculated. One major limitation is that we lack individual-level data on the maternal vitamin A status for most mothers in the trials. Such data would have allowed us to better examine the hypothesis that the efficacy of NVAS is modified by maternal vitamin A status. Although five studies assessed serum retinol in a subset of subjects, due to the small sample sizes for each study there was insufficient power to conduct a subgroup analysis based on these data in two of these five studies. We did consider analysing continuous data in meta-regression but did not take this approach as data points were very few.

Our pooled analyses reveal the absence of an overall benefit of NVAS on mortality, but show substantial heterogeneity in the effect. This heterogeneity is best explained by the characteristics of study populations' contexts, suggesting that NVAS is beneficial in South Asian contexts where the prevalence of maternal VAD is moderate to severe, early infant mortality is high (exceeding 30 deaths per 1000 live births) and maternal schooling is low. On the other hand, no benefit to survival, and even a possibility of harm, is observed in African contexts where maternal VAD is absent or only a mild public health concern, infant mortality is lower, and levels of maternal schooling are higher.

Author group: Neonatal Vitamin A Supplementation Evidence Group

Bangladesh: Keith P West, ${ }^{1}$ Lee S-F Wu, ${ }^{1}$ Hasmot Ali, ${ }^{2}$ Rolf D W Klemm ${ }^{1,3}$ Ghana: Karen Edmond, ${ }^{4}$ Lisa Hurt, ${ }^{5}$ Betty Kirkwood, ${ }^{6}$ Sam Newton, ${ }^{7}$ Caitlin Shannon ${ }^{8}$ India-Haryana: Sunita Taneja, ${ }^{9}$ Sarmila Mazumder, ${ }^{9}$ Kiran Bhatia ${ }^{9}$ Nita Bhandari, ${ }^{9}$ India-Tamil Nadu: Joanne Katz ${ }^{10}$ and James M Tielsch ${ }^{11}$

Indonesia: Jean Humphrey, ${ }^{12}$ Lee S-F Wu, ${ }^{1}$ Tina Agoestina ${ }^{13}$

Pakistan: Sajid Soofi, ${ }^{14}$ Shabina Ariff, ${ }^{14}$ Zaid Bhatti, ${ }^{14}$ Simon Cousens, ${ }^{6}$ Zulfiqar A Bhutta ${ }^{15}$ Zimbabwe: Jean Humphrey ${ }^{12}$ and Robert Ntozini ${ }^{16}$

Tanzania: Honorati Masanja, ${ }^{17}$ Emily R Smith, ${ }^{18}$ Alfa Muhihi, ${ }^{19}$ Wafaie Fawzi ${ }^{18}$

Coordination (WHO): Rajiv Bahl, ${ }^{20}$ Jose Martines, ${ }^{21}$ Sachiyo Yoshida ${ }^{20}$

\section{Author affiliations}

${ }^{1}$ Center for Human Nutrition, Johns Hopkins Bloomberg School of Public Health, Baltimore, Maryland, USA

${ }^{2}$ The JiVitA Project, Johns Hopkins University Bangladesh, Baibandha, Bangladesh ${ }^{3}$ Helen Keller International, New York City, New York, USA

${ }^{4}$ University of Western Australia, Perth, Western Australia, Australia

${ }^{5}$ Cardiff University, Cardiff, UK

${ }^{6}$ London School of Hygiene and Tropical Medicine, London, UK

${ }^{7}$ Kwame Nkrumah University of Science and Technology, Kumasi, Ghana

${ }^{8}$ Engender Health, New York City, New York, USA

${ }^{9}$ Centre for Health Research and Development, New Delhi, Delhi, India

${ }^{10}$ Department of International Health, Johns Hopkins Bloomberg School of Public Health, Baltimore, Maryland, USA

${ }^{11}$ Milken Institute School of Public Health, George Washington University, Washington, DC, USA

${ }^{12}$ Johns Hopkins Bloomberg School of Public Health, Baltimore, Maryland, USA

${ }^{13}$ Hasan Sadikin Hospital, Bandung, Indonesia

${ }^{14}$ Department of Pediatrics and Child Health, The Aga Khan University, Karachi, Sindh, Pakistan

${ }^{15}$ Centre of Excellence in Women and Child Health, The Aga Khan University, Aga Khan, Karachi, Pakistan

${ }^{16}$ Zvitambo Institute for Maternal and Child Health Research, Harare, Zimbabwe

${ }^{17}$ Ifakara Health Institute, Dar es Salaam, Tanzania

${ }^{18}$ Harvard TH Chan School of Public Health, Boston, Massachusetts, USA

${ }^{19}$ Africa Academy for Public Health, Dar es Salaam, Tanzania

${ }^{20}$ Department of Maternal, Newborn, Child and Adolescent Health, World Health Organization, Geneva, Switzerland

${ }^{21}$ CISMAC, Centre for International Health, University of Bergen, Bergen, Norway

Acknowledgements Professor Christine Stabell Benn and the Guinea-Bissau group provided data from their three randomised trials of neonatal vitamin A supplementation (Benn et al 2008, 2010, 2014). Professor Christine Stabell Benn and statistician Andreas Andersen, PhD, participated in the analysis workshops, commented on the paper and qualified for authorship, but declined authorship due to disagreements regarding the methodology and the conclusions. We thank the patients who contributed to the original studies.

Collaborators Neonatal Vitamin A Supplementation Evidence Group

Bangladesh: Keith PWest, Lee S-F Wu, Hasmot Ali, Rolf D W Klemm

Ghana: Karen Edmond, Lisa Hurt, Betty Kirkwood, Sam Newton, Caitlin Shannon India-Haryana: Sunita Taneja, Sarmila Mazumder, Kiran Bhatia, Nita Bhandari, India-Tamil Nadu: Joanne Katz and James M Tielsch Indonesia: Jean Humphrey, Lee S-FWu, Tina Agoestina Pakistan: Sajid Soofi, Shabina Ariff, Zaid Bhatti, Simon Cousens, Zulfiqar A Bhutta Zimbabwe: Jean Humphrey and Robert Ntozini Tanzania: Honorati Masanja, Emily R Smith, Alfa Muhihi, Wafaie Fawzi Coordination (WHO): Rajiv Bahl, Jose Martines, Sachiyo Yoshida

Contributors KPW, LSFW, RDWK, KME, LH, BK, SN, CS, ST, SM, KB, NB, JK, JMT, $J H, S B S, S A, R N, H M, E R S, A M, W F, R B, J M$ and SY participated in the workshop to develop a harmonised pooled analysis protocol and contributed to development of the meta-analysis plan. KH, CS, LSFW, KB, JK, ZAB, SC, RN, ERS and AM analysed the site-specific data. RB, JM and SY consolidated the database and conducted the meta-analysis. ERS wrote the first draft of the manuscript. All authors reviewed, provided the comments and approved the final manuscript.

Funding The meeting of study investigators was organised by the WHO, with financial support from the Bill and Melinda Gates Foundation.

Competing interests JMT received grants from USAID, other from Task Force Sight \& Life, during the conduct of the study. JK received grants from Bill and Melinda Gates Foundation, USAID, and Task Force Sight and Life, during the conduct of the original study contributing to this pooled analysis. KPW received within the past 3 years an award from the Sight and Life Foundation and DSM to support scholarships and academic activities within the programme in Human Nutrition. DSM has prepared gratis nutrient supplement for research.

Patient consent Not required.

Provenance and peer review Not commissioned; externally peer reviewed.

Open access This is an open access article distributed in accordance with the Creative Commons Attribution Non Commercial (CC BY-NC 4.0) license, which permits others to distribute, remix, adapt, build upon this work non-commercially, and license their derivative works on different terms, provided the original work is properly cited, appropriate credit is given, any changes made indicated, and the use is non-commercial. See: http://creativecommons.org/licenses/by-nc/4.0/.

\section{REFERENCES}

1 You D, Hug L, Ejdemyr S, et al. Global, regional, and national levels and trends in under-5 mortality between 1990 and 2015, with scenario-based projections to 2030: a systematic analysis by the UN Inter-agency Group for Child Mortality Estimation. Lancet 2015;386:2275-86.

2 Black RE, Victora CG, Walker SP, et al. Maternal and child undernutrition and overweight in low-income and middle-income countries. Lancet 2013;382:427-51.

3 Imdad A, Mayo-Wilson E, Herzer K, et al. Vitamin A supplementation for preventing morbidity and mortality in children from six months to five years of age. Cochrane Database Syst Rev 2017;3:CD008524.

4 Glasziou PP, Mackerras DE. Vitamin A supplementation in infectious diseases: a metaanalysis. BMJ 1993;306:366-70.

5 Fawzi WW, Chalmers TC, Herrera MG, et al. Vitamin A supplementation and child mortality. A meta-analysis. JAMA 1993;269:898-903

6 Beaton GH, Martorell R, Aronson KJ, et al. Effectiveness of Vitamin A Supplementation in the Control of Young Child Morbidity and Mortality in Developing Countries Nutritionpolicy discussion paper No. 13, 1993.

7 UNICEF. Vitamin A Supplementation: A decade of progress, 2007.

8 Idindili B, Masanja H, Urassa H, et al. Randomized controlled safety and efficacy trial of 2 vitamin A supplementation schedules in Tanzanian infants. Am J Clin Nutr 2007:85:1312-9.

9 Darboe MK, Thurnham DI, Morgan G, et al. Effectiveness of an early supplementation scheme of high-dose vitamin A versus standard WHO protocol in Gambian mothers and infants: a randomised controlled trial. Lancet 2007:369:2088-96.

10 West KP, Katz J, Shrestha SR, et al. Mortality of infants $<6$ mo of age supplemented with vitamin A: a randomized, double-masked trial in Nepal. Am J Clin Nutr 1995;62:143-8.

11 Randomised trial to assess benefits and safety of vitamin A supplementation linked to immunisation in early infancy. WHO/CHD Immunisation-Linked Vitamin A Supplementation Study Group. Lancet 1998:352:1257-63.

12 Humphrey JH, Agoestina T, Wu L, et al. Impact of neonatal vitamin A supplementation on infant morbidity and mortality. J Pediatr 1996;128:489-96.

13 Klemm RD, Labrique AB, Christian P, et al. Newborn vitamin A supplementation reduced infant mortality in rural Bangladesh. Pediatrics 2008;122:e242-50. 
14 Rahmathullah L, Tielsch JM, Thulasiraj RD, et al. Impact of supplementing newborn infants with vitamin A on early infant mortality: community based randomised trial in southern India. BMJ 2003;327:254.

15 Humphrey JH, Iliff PJ, Marinda ET, et al. Effects of a single large dose of vitamin $A$, given during the postpartum period to HIV-positive women and their infants, on child HIV infection, HIV-free survival, and mortality. J Infect Dis 2006;193:860-71.

16 Malaba LC, lliff PJ, Nathoo KJ, et al. Effect of postpartum maternal or neonatal vitamin A supplementation on infant mortality among infants born to HIV-negative mothers in Zimbabwe. Am J Clin Nutr 2005;81:454-60.

17 Benn CS, Fisker AB, Napirna BM, et al. Vitamin A supplementation and BCG vaccination at birth in low birthweight neonates: two by two factorial randomised controlled trial. BMJ 2010;340:c1101.

18 Benn CS, Diness BR, Roth A, et al. Effect of 50,000 IU vitamin A given with BCG vaccine on mortality in infants in Guinea-Bissau: randomised placebo controlled trial. BMJ 2008;336:1416-20.

19 Benn CS, Diness BR, Balde I, et al. Two different doses of supplemental vitamin A did not affect mortality of normal-birth-weight neonates in Guinea-Bissau in a randomized controlled trial. J Nutr 2014;144:1474-9.

20 Haider BA, Bhutta ZA. Neonatal vitamin A supplementation for the prevention of mortality and morbidity in term neonates in developing countries. Cochrane Database Syst Rev 2011;10:CD006980.

21 Gogia S, Sachdev HS. Vitamin A supplementation for the prevention of morbidity and mortality in infants six months of age or less. Cochrane Database Syst Rev 2011; 10:CD007480

22 Mazumder S, Taneja S, Bhatia K, et al. Efficacy of early neonatal supplementation with vitamin A to reduce mortality in infancy in Haryana, India (Neovita): a randomised, double-blind, placebo-controlled trial. Lancet 2015;385.

23 Masanja H, Smith ER, Muhihi A, et al. Effect of neonatal vitamin A supplementation on mortality in infants in Tanzania (Neovita): a randomised, double-blind, placebocontrolled trial. Lancet 2015;385.

24 Edmond KM, Newton S, Shannon C, et al. Effect of early neonatal vitamin A supplementation on mortality during infancy in Ghana (Neovita): a randomised, double-blind, placebo-controlled trial. Lancet 2015;385.

25 Soofi S, Ariff S, Sadiq K, et al. Evaluation of the uptake and impact of neonatal vitamin A supplementation delivered through the Lady Health Worker programme on neonatal and infant morbidity and mortality in rural Pakistan: an effectiveness trial. Arch Dis Child 2017;102:216-23.
26 WHO. Serum retinol concentrations for determining the prevalence of vitamin A deficiency in populations. Geneva: World Health Organization, 2011.

27 WHO. Indicators for assessing vitamin A deficiency and their application in monitoring and evaluating intervention programmes. Geneva: WHO, 1996.

28 WHO. Global prevalence of vitamin A deficiency in populations at risk 1995-2005. WHO Global Database on Vitamin A Deficiency. Geneva: WHO, 2009.

29 Haider BA, Sharma R, Bhutta ZA. Neonatal vitamin A supplementation for the prevention of mortality and morbidity in term neonates in low and middle income countries. Cochrane Database Syst Rev 2017;2:CD006980.

30 West Jr KP. Public health impact of preventing vitamin A deficiency in the first six months of life: Karger Publishers, 2003.

31 Tielsch JM, Rahmathullah L, Thulasiraj RD, et al. Newborn vitamin A dosing reduces the case fatality but not incidence of common childhood morbidities in South India. J Nutr 2007;137:2470-4.

32 Reifen R, Nur T, Ghebermeskel K, et al. Vitamin A deficiency exacerbates inflammation in a rat model of colitis through activation of nuclear factor-kappaB and collagen formation. J Nutr 2002:132:2743-7.

33 Nur T, Peijnenburg AA, Noteborn HP, et al. DNA microarray technology reveals similar gene expression patterns in rats with vitamin a deficiency and chemically induced colitis. J Nutr 2002;132:2131-6.

34 Long KZ, Santos JI, Estrada Garcia T, et al. Vitamin A supplementation reduces the monocyte chemoattractant protein-1 intestinal immune response of Mexican children. J Nutr 2006;136:2600-5.

35 Coles CL, Rahmathullah L, Kanungo R, et al. Vitamin A supplementation at birth delays pneumococcal colonization in South Indian infants. J Nutr 2001;131:255-61.

36 Benn CS, Aaby P, Arts RJ, et al. An enigma: why vitamin A supplementation does not always reduce mortality even though vitamin A deficiency is associated with increased mortality. Int J Epidemiol 2015;44:906-18.

37 Darlow BA, Graham PJ, Rojas-Reyes MX. Vitamin A supplementation to prevent mortality and short- and long-term morbidity in very low birth weight infants. Cochrane Database Syst Rev 2016;22:CD000501.

38 Mactier H, Weaver LT, Vitamin A. Vitamin A and preterm infants: what we know, what we don't know, and what we need to know. Arch Dis Child Fetal Neonatal Ed 2005:90:F103-8.

39 Smith ER, Muhihi A, Mshamu S, et al. The effect of neonatal vitamin A supplementation on morbidity and mortality at 12 months: a randomized trial. Int $J$ Epidemiol 2016;45:2112-21. 